\title{
A produção científica do Rio Grande do Sul em Ciências Agrárias representada na base Web of Science
}

\author{
The scientific production of Rio Grande do Sul in the Agricultural Sciences \\ represented in the database Web of Science
}

\author{
Rosely de Andrade Vargas $^{\mathrm{I}^{*}}$ Samile Andréa de Souza Vanz ${ }^{\mathrm{I}}$
}

RESUMO

Este trabalho caracteriza a produção científica do Rio Grande do Sul em Ciências Agrárias representada na base de dados Web of Science de 2000 a 2010. A partir da análise de 6.617 registros, feita com o auxílio do software Bibexcel, são identificados documentos, idiomas e revistas mais utilizadas pelos pesquisadores gaúchos na divulgação de suas investigações. Apresenta-se também a evolução anual da produção e as instituições de ensino e pesquisa mais produtivas. Os resultados mostram que a produção científica do Estado em Ciências Agrárias indexada na base registrou forte crescimento, ascendendo de 179 documentos em 2000 para 1.107 documentos em 2010. Os artigos científicos corresponderam a 92\% do total das publicações. Inglês e português foram os idiomas mais usados. Entre os 20 periódicos com maior volume de publicações, 18 são do Brasil. A revista Ciência Rural liderou o ranking dos periódicos mais utilizados, concentrando $10 \%$ do total da produção no período.

Palavras-chave: ciências agrárias, bibliometria, indicadores de produção científica.

\section{ABSTRACT}

This paper characterizes the scientific output of the state of Rio Grande do Sul in agricultural sciences as represented in the database Web of Science between 2000-2010. Based on the analysis of 6,617 indexed documents, using Bibexcel, we identified types of documents, languages and journals most frequently used by authors to disseminate their research. The annual production growth and a list of the most productive institutions are also presented. The results reveal that agricultural research in Rio Grande do Sul, indexed in the Web of Science, showed a strong growth, increasing from 179 articles in 2000 to 1,107 documents in 2010. Scientific papers accounted for $92 \%$ of the publications. English and Portuguese were the most used languages by the authors. As to the journals, 18 out of the 20 titles most used by researchers are published in Brazil. The journal Ciência Rural led the ranking of the most used journals, accounting $10 \%$ of total output during 2000-2010.
Key words: agricultural sciences, bibliometrics, scientific indicators.

\section{INTRODUÇÃO}

A avaliação quantitativa da ciência, feita a partir de estudos bibliométricos baseados em dados extraídos das publicações científicas, vem sendo incentivada em vários países devido ao aumento do volume de investigações e ao acirramento da competição por recursos entre regiões e instituições de ensino e pesquisa. No Brasil, o interesse pelos estudos bibliométricos disseminou-se em especial nos últimos anos, quando as agências de fomento apropriaram-se de indicadores de produtividade (número de publicações) e de visibilidade (número de citações) para estimar o desempenho da ciência nacional e dar suporte a modelos de avaliação individuais e institucionais, como no caso dos programas de pós-graduação (MUGNAINI et al., 2004; VANZ \& STUMPF, 2010; LETA 2011).

Como uma das áreas mais tradicionais da ciência brasileira, as Ciências Agrárias têm sido objeto de importantes estudos bibliométricos nos últimos anos. Entre as investigações mais abrangentes, destaca-se a de LYRA \& GUIMARÃES (2007). Com base em registros bibliográficos de publicações indexadas na Standard Data Base e na Deluxe Data Base, do Institute for Scientific Information (ISI), eles compararam a produção nacional com a mundial, de 1981 a 2006, e constaram que o Brasil ocupou a $12^{\text {a }}$

IPrograma de Pós-graduação em Comunicação e Informação, Universidade Federal do Rio Grande do Sul (UFRGS), 90035-007, Porto Alegre, RS, Brasil. E-mail: vargasrosely@yahoo.com.br. *Autor para correspondência. 
posição no ranking mundial, apresentando resultados superiores aos obtidos por países de economia emergente, como a Coreia do Sul e a Rússia. Apesar do expressivo volume de publicações brasileiras na área, os autores consideraram o resultado "modesto diante da relevância da agropecuária na economia e do seu papel na balança comercial do país” (LYRA \& GUIMARÃES, 2007).

PENTEADO FILHO \& ÁVILA (2009) empregaram técnicas bibliométricas para avaliar a participação da Embrapa em artigos de periódicos indexados na Web of Science de 1977 a 2006. Os autores identificaram as revistas que mais publicaram artigos da Embrapa e as parcerias das instituições que realizaram trabalhos com a empresa. Constataram que a Embrapa destacouse, no período, como uma das dez instituições brasileiras com maior número de artigos na base de dados.

O presente estudo utiliza técnicas bibliométricas com o objetivo de caracterizar a produção gaúcha em Ciências Agrárias. Para tanto, foram analisadas publicações de autores vinculados a instituições de ensino e pesquisa do Estado, indexadas na Web of Science de 2000 a 2010. Entre outros indicadores, são apresentados idiomas, tipos de documentos e periódicos mais utilizados pelos autores para publicação, assim como instituições de ensino e pesquisa mais produtivas. O trabalho integra um projeto mais amplo, intitulado “A ciência no Rio Grande do Sul: mapeamento da produção e colaboração nos anos de 2000 a 2011”, elaborado pelo Grupo de Pesquisa em Comunicação Científica da Universidade Federal do Rio Grande do Sul (UFRGS) e realizado com o apoio da Fundação de Amparo à Pesquisa do Estado do Rio Grande do Sul (FAPERGS).

O interesse pela área se dá em razão de sua importância na história e na economia gaúcha. A primeira escola superior de Veterinária do Brasil, a Imperial Escola de Medicina Veterinária e de Agricultura Prática, foi criada em Pelotas em 1883 (BRASIL, 1884; CAPDEVILLE, 1991). No século XX, entre o final dos anos 60 e início dos anos 70, começaram a funcionar, na Universidade Federal do Rio Grande do Sul (UFRGS) e na Universidade Federal de Santa Maria (UFSM), os primeiros programas de pós-graduação em Ciências Agrárias do estado, que têm seu Produto Interno Bruto (PIB) atrelado ao setor agropecuário. O estudo pode, portanto, contribuir para dar uma visão do estágio da pesquisa gaúcha em Ciências Agrárias na última década.

\section{MATERIAL E MÉTODOS}

A pesquisa tem abordagem quantitativa com perspectiva bibliométrica. Como principal fonte, utilizou-se a Web of Science, base de dados multidisciplinar da Thomson Reuters que reúne mais de 36 milhões de registros. O corpus da pesquisa é formado por publicações de Ciências Agrárias indexadas no Science Citation Index Expanded (SCIE) e no Social Sciences Citation Index (SSCI), de 2000 a 2010, com, pelo menos, um endereço do Rio Grande do Sul no campo Author Address.

O trabalho foi realizado em duas etapas. Inicialmente, fez-se a coleta de registros na opção de busca avançada da base. A expressão empregada foi: CU=(Brasil OR Brazil) AND PS=(RS OR Rio Grande do Sul). Para maior recuperação, foram feitas buscas avançadas, também no campo Author Address, com nomes de instituições de ensino e pesquisa do Estado. Foram coletados todos os tipos de documentos - anais, artigos científicos, cartas, correções, editoriais, resumos de eventos, revisões, etc. Após a retirada de dados duplicados, obteve-se um arquivo com 29.560 registros.

$\mathrm{Na}$ segunda etapa, a partir do esquema de classificação de assuntos de GLÄNZEL \& SCHUBERT (2003), ajustado às categorias de assunto da base de dados e à tabela de áreas de conhecimento da Coordenação de Aperfeiçoamento de Pessoal de Nível Superior (CAPES), fez-se a extração de dados das Ciências Agrárias do arquivo com 29.560 documentos. Quando da seleção de assuntos, decidiu-se não incluir a categoria Multidisciplinary Science na lista de assuntos, pois sua inclusão aumentaria o volume de dados, exigindo mais tempo para extração de registros não concernentes à pesquisa agrícola. Em função disso, publicações da revista Ciência Rural, na época, classificada na base nesta categoria, foram anexados manualmente ao arquivo das Ciências Agrárias. Obtevese assim um arquivo com 6.617 registros.

Para organização e tratamento dos dados, usou-se o Bibexcel, software de domínio público, criado por Olle Person, da Universidade Umea (Suécia), largamente empregado em pesquisas bibliométricas, que agrupa as informações dos diversos campos dos registros bibliográficos. As análises estatísticas foram feitas com o aplicativo Microsoft Office Excel Windows ${ }^{(\mathrm{tm})}$, versão 2007 (Microsoft, Redmond, EUA).

\section{RESULTADOS E DISCUSSÃO}

Entre 2000 e 2010, pesquisadores de Ciências Agrárias vinculados a instituições do Estado 
tiveram 6.617 trabalhos indexados no SCIE e no SSCI. A produção anual, que somava 179 publicações em 2000, subiu para 1.107 publicações em 2010, com alta de 518,4\% no período. Os resultados confirmam a tendência de forte crescimento da produção na grande área verificada, em termos nacionais, por ADAMS \& KING (2009) e LETA (2012). As maiores taxas de crescimento foram registradas em 2003 (39\%) e em 2008 (73\%). Entre 2009 e 2010, houve uma leve queda no número de publicações, ocasionada pelo fato de que os artigos de 2010 haviam sido indexados parcialmente pela base até a coleta de dados, feita em 19 janeiro de 2012. Apesar da queda, o crescimento da produção gaúcha em Ciências Agrárias ajusta-se bem à linha de crescimento exponencial com $\mathrm{R}^{2}=$ 0,9598 (Figura 1).

Ao que parece, as altas taxas de crescimento em 2003 e 2008, estão relacionadas com a inserção de periódicos nacionais na base. Em 2003, a Revista Brasileira de Ciência do Solo, publicada pela Sociedade Brasileira de Ciência do Solo (SBCS) e a Ciência e Agrotecnologia, publicada pela Universidade Federal de Lavras (UFLA), passaram a ser indexadas na Web of Science. Já entre 2007 e 2008, dezenas de revistas nacionais foram inseridas na base. No conjunto, constavam títulos editados no Estado, como a Acta Scientiae Veterinariae, publicada pela
UFRGS, Ciência Florestal e Ciência Rural, publicadas pela Universidade Federal de Santa Maria (UFSM), e Iheringia Série Zoologia, da Fundação Zoobotânica.

Conforme TESTA (2010), a ampliação mais expressiva no conjunto de títulos nacionais indexados na Web of Science envolveu a categoria Agriculture Biology \& Environmental Sciences (ABES). Até 2005, a base reunia 11 periódicos do Brasil nesses campos. A partir de 2005, foram incluídos 34 novos títulos. A partir daí, como ressalta PACKER (2011), a base da Thomson Reuters passou a indexar um conjunto maior de periódicos brasileiros das Ciências Agrárias do que o conjunto indexado pela Scientific Electronic Library Online (Scielo). Segundo PACKER (2011),a ampliação ajudou "a elevar o Brasil da 17a para a 13" posição no ranking da produção científica mundial, medida pela base em 2009”.

Outro fator que ajudou a elevar as taxas anuais de crescimento do número de publicações foi o encurtamento dos prazos de periodicidade das revistas. Muitos periódicos nacionais da área agrícola reduziram o intervalo de tempo entre a publicação de seus fascículos nos últimos anos. Com isso, mais artigos passaram a ser publicados e indexados.

A expansão dos programas de pósgraduação (PPGs) em Ciências Agrárias no Estado - de 21 programas em 2000 para 29 programas em

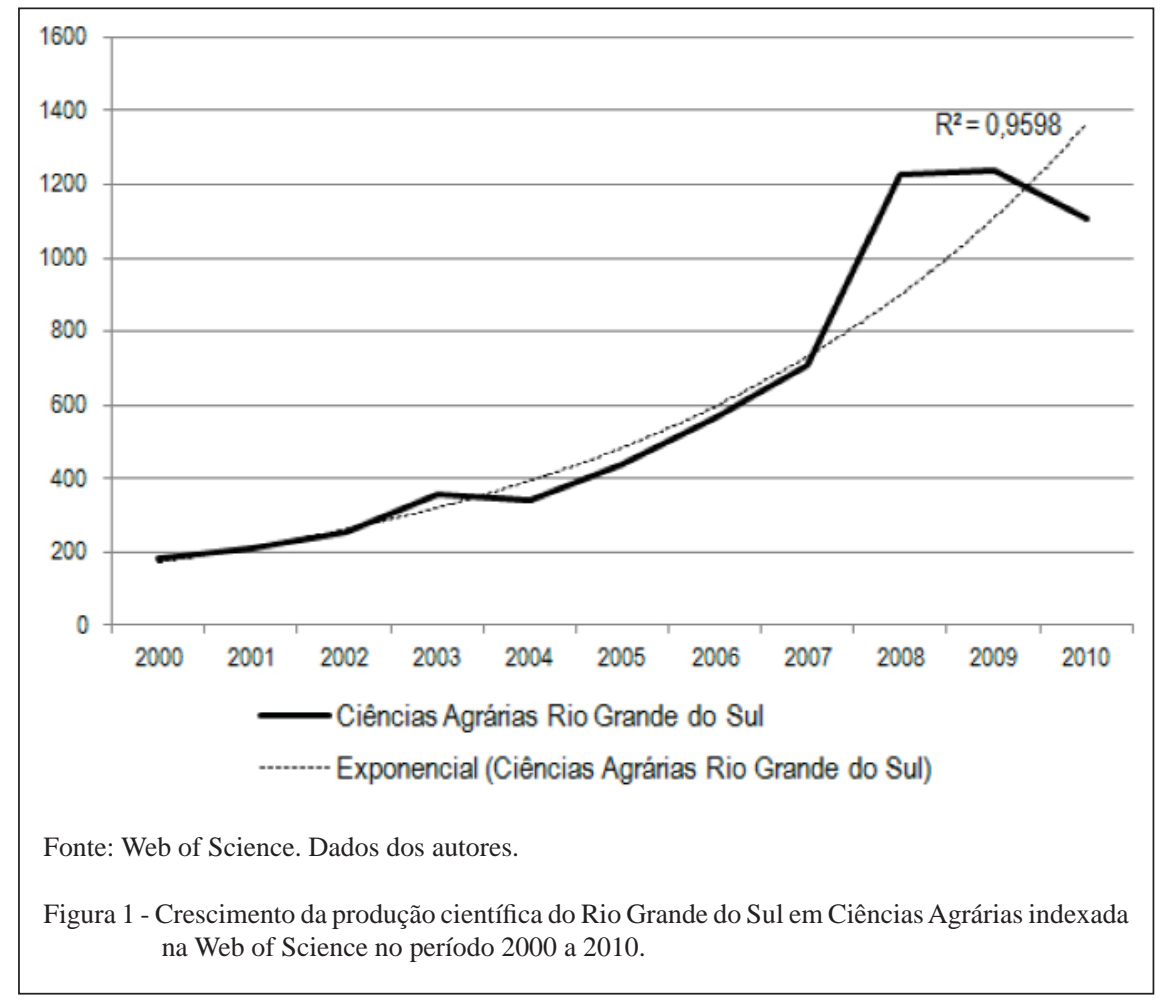

Ciência Rural, v.44, n.5, mai, 2014. 
2010 - e o consequente aumento do número de alunos em cursos de mestrado e doutorado também devem ser considerados na avaliação do crescimento das publicações. O total de alunos nos PPGs duplicou no período, mas é a partir do ingresso de periódicos do Rio Grande do Sul na Web of Science, entre 2007 e 2008, que o crescimento da produção científica gaúcha indexada na base de dados torna-se mais expressivo (Figura 2).

Entre os 6.617 documentos que compõem essa produção, 92\% são artigos, indicando que o artigo em periódico científico avaliado pelos pares mantém-se como principal forma de comunicação empregada pelos pesquisadores da área para difundir os resultados de suas investigações (MEADOWS, 1999). O segundo tipo de documento mais utilizado é o resumo de evento, com 3,5\%, seguido por anais, com 2,5\%. Cartas, material editorial e notas compuseram os demais $2 \%$ do total.

Quanto ao idioma, 61\% das publicações estão em inglês, o que confere visibilidade internacional aos trabalhos (MEADOWS, 1999). Entretanto, merece destaque o alto percentual (38\%) de registros em português. O resultado, que também está relacionado com a inserção de maior número de revistas do Brasil na base de dados, evidencia uma característica da produção nacional em Ciências Agrárias (ARVANITIS \& CHATELIN, 1994). Como explica VELHO (2008), a agenda de pesquisa aplicada "de caráter nacional, local e multidisciplinar, que define os contornos do público-alvo para os resultados produzidos" contribui para que parte da pesquisa agrícola brasileira seja escrita em português e publicada em periódicos nacionais. Já o número de artigos em espanhol, apenas 47, é modesto quando se leva em conta as fronteiras do Estado com a Argentina e o Uruguai e os programas de integração decorrentes do Mercosul.

Foram identificadas 744 revistas com artigos de autores gaúchos. Porém, metade da produção concentra-se num conjunto de 20 títulos entre os quais 18 são editados no Brasil. Os resultados sobre as revistas mais utilizadas para publicação (Tabela 1) reiteram as afirmações de VELHO (2008) sobre a preferência dos pesquisadores da área pelas publicações em português, em periódicos nacionais.

A revista Ciência Rural, editada pelo Centro de Ciências Rurais da UFSM, concentra o

Tabela 1 - Periódicos indexados na Web of Science com maior número de publicações de autores do Rio Grande do Sul na área de Ciências Agrárias no período 2000-2010.

\begin{tabular}{|c|c|c|c|}
\hline Periódico & $\mathrm{n}^{\mathrm{o}}$. artigos & $\%$ & País \\
\hline Ciência Rural & 619 & 10,2 & Brasil \\
\hline Revista Brasileira de Zootecnia & 368 & 6,0 & Brasil \\
\hline Pesquisa Agropecuária Brasileira & 355 & 5,8 & Brasil \\
\hline Revista Brasileira de Ciência do Solo & 248 & 4,1 & Brasil \\
\hline Pesquisa Veterinária Brasileira & 240 & 3,9 & Brasil \\
\hline Acta Scientiae Veterinariae & 168 & 2,8 & Brasil \\
\hline Arquivo Brasileiro de Medicina Veterinária e Zootecnia & 152 & 2,5 & Brasil \\
\hline Brazilian Journal of Microbiology & 123 & 2,0 & Brasil \\
\hline Brazilian Journal of Medical and Biological Research & 105 & 1,7 & Brasil \\
\hline Revista Brasileira de Fruticultura & 104 & 1,7 & Brasil \\
\hline Planta Daninha & 104 & 1,7 & Brasil \\
\hline Journal of Coastal Research & 103 & 1,7 & EUA \\
\hline Brazilian Archives of Biology and Technology & 102 & 1,7 & Brasil \\
\hline Zootaxa & 101 & 1,7 & Nova Zelândia \\
\hline Iheringia Serie Zoologia & 91 & 1,5 & Brasil \\
\hline Ciência Florestal & 83 & 1,4 & Brasil \\
\hline Ciência e Tecnologia de Alimentos & 78 & 1,3 & Brasil \\
\hline Ciência e Agrotecnologia & 67 & 1,1 & Brasil \\
\hline Revista Brasileira de Zoologia & 64 & 1,0 & Brasil \\
\hline Scientia Agricola & 59 & 1,0 & Brasil \\
\hline Subtotal 20 revistas & 3.334 & 54,8 & \\
\hline Demais periódicos & 2.753 & 45,20 & \\
\hline Total de artigos indexados & 6.087 & 100,00 & \\
\hline
\end{tabular}

Fonte: Web of Science. Dados dos autores. 
maior número de registros, 10,16\% do total de 6.087 artigos. Em segundo lugar, está a Revista Brasileira de Zootecnia, publicada pela Sociedade Brasileira de Zootecnia. A Pesquisa Agropecuária Brasileira, publicada pela Embrapa, ocupa o terceiro lugar, seguida pela Revista Brasileira de Ciência do Solo, publicação da Sociedade Brasileira de Ciência do Solo.

Os dois primeiros periódicos, que concentraram a maior parte da produção gaúcha em Ciências Agrárias, também são citados por LETA (2012) em recente estudo sobre o crescimento da produção científica nacional indexada na Web of Science e na Scopus. Conforme levantamento feito pela autora, a Ciência Rural ficou na primeira posição no ranking de revistas com maior número de publicações brasileiras, em 2010, nas duas bases de dados, considerando todas as áreas de conhecimento. A Revista Brasileira de Zootecnia, por sua vez, ocupou a terceira posição na Scopus e a quinta na Web of Science. Para LETA (2012), a projeção da ciência brasileira no cenário internacional nos últimos anos deu-se principalmente em função da maior qualificação dos recursos humanos envolvidos na atividade de pesquisa e da ampliação do conjunto de periódicos nacionais nas duas bases de dados.

$\mathrm{Na}$ análise sobre instituições com maior número de publicações, a UFRGS aparece em destaque, com 2.575 documentos, cerca de $40 \%$ do total da produção. Oresultado confirma a proeminência da universidade como uma das instituições do país com maior número de publicações indexadas na Web of Science (FAPESP, 2011). A UFSM, com 1.841 publicações foi responsável pela segunda maior contribuição. Em terceiro lugar aparece a UFPel, com 818 publicações, seguida pela Embrapa com 774 publicações.

Os dados sobre o desempenho das instituições de ensino e pesquisa revelam que a pesquisa gaúcha na área desenvolve-se de forma descentralizada. Há pelo menos quatro importantes polos de pesquisa instalados na Grande Porto Alegre e nas regiões central, sul e noroeste. Os investigadores desses polos trabalham em colaboração com instituições locais, nacionais e estrangeiras. As principais instituições nacionais que atuam com instituições do Rio Grande do Sul são a Universidade de São Paulo (USP), Universidade Federal de Viçosa (UFV) e Universidade Estadual Paulista Julio de Mesquita Filho (UNESP). As parcerias internacionais mais importantes são desenvolvidas com a Universidade da Flórida, o Serviço de Pesquisas Agrícolas do Departamento de Agricultura dos Estados Unidos (USDA-ARS) e a Universidade de Wisconsin (EUA). A estreita relação com os Estados Unidos é antiga: foi a partir de intercâmbio com instituições estadunidenses, como a Universidade de Wisconsin, que surgiram no Estado os primeiros cursos de pós-graduação na área de Agronomia (ALMEIDA \& MACHADO, 2009). Já na área de Medicina Veterinária, há parcerias com instituições da Alemanha, como a Escola Superior de Veterinária de Hannover e Univerdade de Freiburg, entre outras. A escola de Hannover colaborou, com o envio de profissionais e a doação de equipamentos, para a criação dos primeiros cursos de pós-graduação do estado na área de Veterinária (UFRGS, 2009).

\section{CONCLUSÃO}

O desempenho positivo da produção gaúcha em Ciências Agrárias segue tendência verificada em estudos anteriores, que apontam a grande área como um dos campos que mais tem contribuído para projeção da ciência brasileira em bases de dados internacionais (MEIS et al., 2007; VANZ, 2009; FAPESP, 2011). Nesses estudos, a expansão do número de PPGs e o aumento dos investimentos em pesquisa são apontados como fatores determinantes para o volume crescente de pesquisas e de publicações nesta e em outras grandes áreas de conhecimento.

No caso específico da produção gaúcha, como se destacou, observou-se um aumento considerável do número de alunos nos PPGs em Ciências Agrárias. Ao lado de São Paulo e do Rio de Janeiro, o Rio Grande do Sul foi um dos estados que recebeu mais recursos do Conselho Nacional de Desenvolvimento Científico e Tecnológico (CNPq) entre 2006 e 2008 (FAPESP, 2011). A Coordenação de Aperfeiçoamento de Pessoal de Nível Superior (CAPES), por sua vez, ampliou o número de bolsas de pós-graduação para a grande área das Ciências Agrárias no Estado: entre 2000 e 2010, o número de bolsas para cursos de doutorado aumentou 300\%. Para cursos de mestrado, o aumento foi de $95 \%$ (CAPES, 2013).

A par da expansão dos PPGs e dos investimentos, dois fatores pontuais contribuíram de forma significativa para elevar o número de publicações do Rio Grande Sul em Ciências Agrárias na base de dados entre 2000 e 2010: a ampliação do conjunto de revistas nacionais na Web of Science e o encurtamento dos prazos de periodicidade de várias dessas revistas. Com a ampliação, títulos editados em núcleos de excelência do Estado, como 


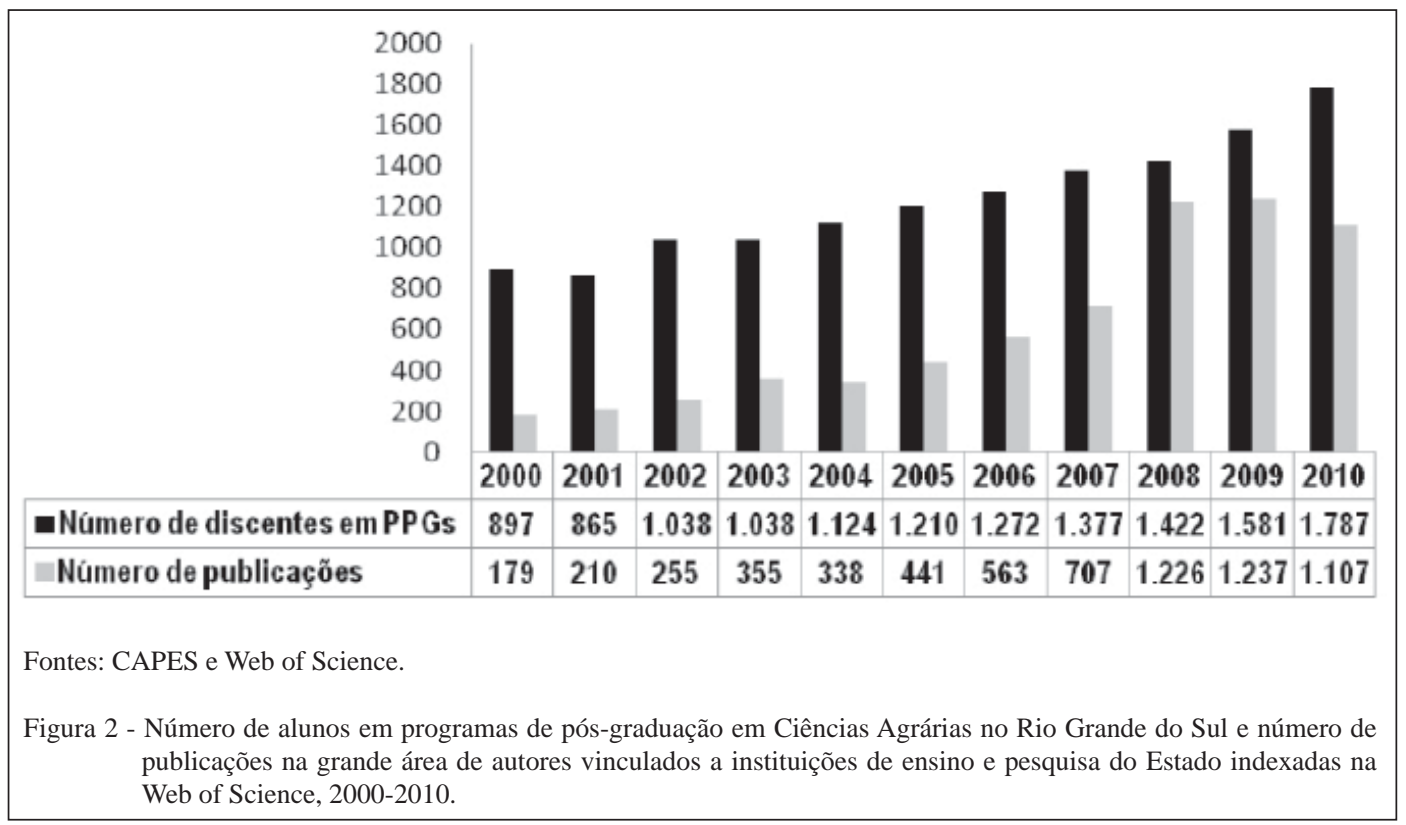

UFRGS e UFSM, começaram ser indexados na base. Paralelamente, muitas revistas da grande área alteraram sua periodicidade.

A Pesquisa Veterinária Brasileira, editada pelo Colégio Brasileiro de Patologia Animal (CBPA), trimestral desde a sua criação em 1981, adotou periodicidade mensal em 2007. A Revista Brasileira de Zootecnia e a Ciência Rural, antes bimestrais, também adotaram periodicidade mensal, respectivamente, em 2008 e 2010. A Revista Brasileira de Fruticultura e a Acta Scientiae Veterinariae, quadrimestrais, passaram a ter periodicidade trimestral, respectivamente, em 2008 e 2009. Ou seja, cinco periódicos, entre os dez títulos mais usados para publicação pelos autores do Rio Grande do Sul, reduziram o intervalo de tempo entre a publicação de seus fascículos nos últimos anos. Provavelmente relacionada com o processo de aperfeiçoamento das revistas, esta mudança garantiu mais espaço para a divulgação dos resultados de pesquisas, impulsionando a produção científica gaúcha em Ciências Agrárias indexada na base internacional no período 2000-2010.

A pesquisa integra um projeto maior em desenvolvimento, que visa a mapear a produção científica do Rio Grande do Sul na última década. Os resultados da área de Ciências Agrárias, aqui apresentados, abrem caminho para futuras investigações, ainda mais abrangentes, com o acréscimo de novas fontes de informação, como as bases de dados Scopus e Scielo, e a inclusão de análises sobre citação e impacto das publicações gaúchas em Ciências Agrárias durante o período.

\section{REFERÊNCIAS}

ADAMS, J.; KING, C. Global research report: Brazil - Research and collaboration in the new geography of science. Leeeds : Thomson Reuters, 2009. 12p.

ALMEIDA, J.; MACHADO, J. (Orgs.). Desenvolvimento Rural no Cone Sul - Desarrollo rural en el Cono Sur. Porto Alegre : Associação Holos, 2009. 365p.

ARVANITIS, R.; CHATELIN, Y. Bibliometrics of tropical soil sciences: some reflections and orientations. In: McDONALD, P. The literature of soil science. Ithaca: Cornell University, 1994. p.73-94.

BRASIL. Ministério da Agricultura, Comércio e Obras Públicas. Relatório ministerial. Rio de Janeiro: Imprensa Nacional, 1884. Disponível em: <http://www.crl.edu/brazil/ministerial/ agricultura>. Acesso em: 02 ago. 2012.

CAPDEVILLE, G. O ensino superior agrícola no Brasil. Viçosa: UFV, 1991. 184p.

CAPES (COORDENAÇÃO DE APERFEIÇOAMENTO DE PESSOAL DE NÍVEL SUPERIOR). Estatísticas. Brasília: CAPES, 2006. Disponível em: <http://www.capes.gov.br/estatisticas $>$. Acesso em 20 jul. 2013.

FAPESP. Análise da produção científica a partir de publicações em periódicos especializados. In: __ . Indicadores de ciência, tecnologia e inovação em São Paulo 2010. São Paulo: FAPESP, 2011. Cap. 4, p.15-24.

GLÄNZEL, W.; SCHUBERT, A. A new classification scheme of science fields and subfields designed for scientometric evaluation purposes. Scientometrics, Dordrecht, v.56, n.3, p.357-367, 2003.

LETA, J. Indicadores de desempenho, ciência brasileira e a cobertura das bases informacionais. Revista USP, São Paulo, n.89, p.62-77, 2011. Disponível em <http://rusp.scielo.br/scielo.

Ciência Rural, v.44, n.5, mai, 2014. 
php?script=sci_arttext\&pid=S0103-99892011000200005\&lng=pt \&nrm=iso>. Acesso em: 13 ago. 2013.

LETA, J. Brazilian growth in the mainstream science: The role of human resources and national journals. Journal of Scientometric Research, Mumbai, v.1, n.1, p.44-52, 2012.

LYRA, T.; GUIMARÃES, J.A. Produção Cientifica brasileira em comparação com o desempenho mundial em Ciências Agrárias. Planejamento e Políticas Públicas, Brasília, v.30, p.5-15, 2007. Disponível em: <http://www.ipea.gov.br/ppp/index.php/PPP/ article/view/39>. Acesso em: 20 set. 2013.

MEADOWS, A.J. A comunicação científica. Brasília: Briquet de Lemos, 1999. 268p.

MEIS, L. et al. The impact of science in Brazil. IUBM Life, London, v.59, n.4-5, p.227-234, 2007.

MUGNAINI, R. et al. Indicadores bibliométricos da produção científica brasileira: uma análise a partir da base Pascal. Ciência da Informação, Brasília, v.33, n.2, p.123-131, 2004. Disponível em: $<$ http://www.scielo.br/scielo.php?script=sci_arttext\&pid=S010019652004000200013\&lng=en\&nrm=iso $>$. Acesso em: $10 \mathrm{jul}$. 2013.

PACKER, A.L. Os periódicos brasileiros e a comunicação da pesquisa nacional. Revista USP, São Paulo, n.89, p.26-61, 2011. Disponível em: <http://rusp.scielo.br/scielo.php?script=sci_ arttext\&pid=S0103-99892011000200004\&lng=en\&nrm=iso $>$. Accesso em: 13 jul. 2013.
PENTEADO FILHO, R.; ÁVILA, A. Embrapa Brasil: análise bibliométrica dos artigos na Web of Science (1977-2006). Brasília: Embrapa, 2009. Disponível em: <http://www.embrapa. br/publicacoes/tecnico/folderTextoDiscussao/arquivos-pdf/ Texto-36_05-11-09.pdf>. Acesso em: 10 dez. 2012.

TESTA, J. The Globalization of Web of Science: 2005-2010. New York: Thomson Reuters, 2011. Disponível em: <http://wokinfo. com/media/pdf/globalwos-essay.pdf>. Acesso em: 10 abr. 2013.

UFRGS. 40 anos do Programa de Pós-graduação em Ciências Veterinária. Porto Alegre: UFRGS, 2009. 103p. Disponível em: <http://www.ufrgs.br/ppgcv/o-programa/historico-1/historico/ at_download/file>. Acesso em: 20 set. 2013.

VANZ, S.A.S; STUMPF, I.R.C. Procedimentos e ferramentas aplicados aos estudos bibliométricos. Informação \& Sociedade, João Pessoa, v.20, p.65-75, 2010. Disponível em: <http://www.ies.ufpb.br/ojs/index. php/ies/article/view/4817>. Acesso em: 11 nov. 2012.

VANZ, S.A.S. As redes de colaboração científica no Brasil: 2004-2006. 2009. 204f. Tese (Doutorado em Comunicação e Informação) - Programa de Pós-graduação em Comunicação e Informação, Universidade Federal do Rio Grande do Sul, RS. Disponível em: <http://www.lume.ufrgs.br/handle/10183/17169>. Acesso em: 10 mar. 2012.

VELHO, L. Publicação científica e avaliação nas Ciências Agrárias: pontos para discussão. Boletim Informativo da Sociedade Brasileira de Ciência do solo, Viçosa: Sociedade Brasileira de Ciência do Solo, 2008. p.22-24. 